\title{
周Thieme
}

\section{Vaginale Blutung als Symptom eines internistischen Notfalls - Tücken der Therapie mit neuen oralen Antikoagulanzien}

Fall 5/2014 25.7.2014

Nephrologie Gynäkologie

Vaginal bleeding as symptom of a medical emergency pitfalls of therapy with new oral anticoagulants

Dorothea Baumann1, Michael Haap1, Christos Tsaousidis², Bernhard Krämer², Jürgen Schreieck1, Karl Jaschonek ${ }^{1}$, Ingo Rettig ${ }^{1}$, Andreas Peter ${ }^{1}$, Diethelm Wallwiener ${ }^{2}$, Ferruh Artunc ${ }^{1}$

${ }^{1}$ Medizinische Klinik, Universitätsklinikum Tübingen

2 Frauenklinik, Universitätsklinikum Tübingen 
DMW

\section{Falldatenbank}

\section{Einleitung}

> Vaginale Blutungen treten physiologischerweise als Menses auf. Unter pathologischen Bedingungen findet man vaginale Blutungen bei hormonellen Störungen, gutartigen Erkrankungen des Uterus (Myome oder Polypen) oder im Rahmen einer Schwangerschaft. Postmenopausale Blutungen sind als Malignom-suspekt zu werten.

> Vaginale Blutungen verlaufen bei Patientinnen mit angeborenen oder erworbenen Gerinnungsstörungen typischerweise verstärkt und können als erstes Symptom auf eine derartige Störung hindeuten.

> Im hier vorgestellten Fall erwies sich eine vaginale Blutung als gynäkologisches Symptom eines internistischen Notfalls, das durch die Einnahme eines neuen oralen Antikoagulanz aggraviert wurde. 
DMW

\section{Falldatenbank}

\section{Kasuistik | Anamnese}

> Übernahme einer 90-jährigen Patientin auf die internistische Intensivstation aus der Frauenklinik

$>$ Zuvor hatte sich die Patientin in einem heimatnahen Krankenhaus bei spontan aufgetretener vaginaler Blutung vorgestellt.

$>$ Dort wurde vor Verlegung notfallmäßig eine vaginale Tamponade eingelegt.

$>\quad$ Vor 2 Monaten wurde mittels Abrasio ein Endometriumkarzinom diagnostiziert. Die geplante Hysterektomie war bisher noch nicht erfolgt. 


\section{Kasuistik | Vorerkrankungen}

> Die Patientin hatte zahlreiche Komorbiditäten, insbesondere mit kardialem Schwerpunkt:

$>$ Zweifache Synkope infolge eines symptomatischen Sick-Sinus-Syndroms

- Schrittmacherimplantation (DDD) vor 8 Jahren

- Linksschenkelblock

> Vorderwandinfarkt vor 35 Jahren mit leicht reduzierter linksventrikulärer Pumpfunktion

$>$ Leichtgradige Aortenklappenstenose

$>$ Intermittierendes Vorhofflimmern

$>$ Weitere Diagnosen waren

$>$ Lungenarterienembolie bei tiefer Beinvenenthrombose rechts vor 11 Jahren

$>$ Chronische Niereninsuffizienz im Stadium 3

$>$ V.a. Myelodysplastisches Syndrom (MDS) bei Panzytopenie

$>$ Chronisch obstruktive Lungenerkrankung (COPD)

$>$ Anaphylaxie mit konsekutiver Reanimation bei multiplen Nahrungsmittelallergien 


\section{Kasuistik | Medikation vor Aufnahme}

$>$ Bisoprolol $5 \mathrm{mg}$

$>$ Candesartan $16 \mathrm{mg}$

$>$ Digitoxin $0,1 \mathrm{mg}$

> Dabigatran $110 \mathrm{mg}$

$>$ Therapiebeginn zwei Wochen vor Eintreten der vaginalen Blutung, letzte Einnahme am Abend vor Vorstellung in der heimatnahen Klinik; zuvor Therapie mit Phenprocoumon

> Spironolacton $50 \mathrm{mg}$

$>$ Torasemid $10 \mathrm{mg}$
1-0-1

$1 / 2-0-0$

1-0-0

1-0-1

1-0-0

1-1-0 
DMW

\section{Falldatenbank}

UNIVERSITÄTS

KLINIKUM

TÜBINGEN

\section{Kasuistik | Körperlicher Untersuchungsbefund}

$>$ Allgemein

> 90 Jahre, reduzierter Allgemein- und kachektischer Ernährungszustand,

Gewicht bei Aufnahme anamnestisch 60 kg; Größe anamnestisch 168 cm; BMI 21 kg/m²

$>$ ausgeprägter Foetor uraemicus

$>$ trockene Haut, reduzierter Hautturgor

$>$ Cor

$>$ Herztöne leise, rein, rhythmisch, Herzfrequenz 60/min, Blutdruck 100/55 mmHg, Schrittmacheraggregat links pectoral sichtbar, kein Perikardreiben

$>$ Pulmo

$>$ vesikuläres Atemgeräusch, seitengleich belüftet, keine Nebengeräusche bds.

$>$ Abdomen

$>$ Bauchdecke weich, Darmgeräusche regelrecht über allen Quadranten

$>$ Extremitäten

$>$ seitengleich hinsichtlich Umfang und Temperatur, keine Ödeme

$>$ Neurologischer Status

> Hirnnerven o.B., kein fokalneurologisches Defizit; Orientierung zur Zeit unscharf, sonst uneingeschränkt 


\section{Falldatenbank}

Tübinger Fälle

\section{Kasuistik | Diagnostik | Labor bei Aufnahme}

\begin{tabular}{|c|c|c|c|c|c|}
\hline Parameter & Wert & Referenzbereich & Parameter & Wert & Referenzbereich \\
\hline Leukozyten $[1 / \mu l]$ & 10180 & $4100-11800$ & Natrium [mmol/l] & 136 & $136-148$ \\
\hline Hämoglobin [g/dl] & 9,9 & $12,0-16,0$ & Kalium [mmol/l] & 6,3 & $3,5-4,8$ \\
\hline $\begin{array}{l}\text { Mittleres korpuskuläres Hämoglobin } \\
(\mathrm{MCH})[\mathrm{pg}]\end{array}$ & 30,1 & $27-34$ & Kalzium [mmol/l] & 2,6 & $2,1-2,3$ \\
\hline $\begin{array}{l}\text { Mittleres korpuskuläres Volumen } \\
\text { (MCV) [fl] }\end{array}$ & 87,3 & $80-93$ & Phosphat [mmol/l] & 2,4 & $0,8-1,5$ \\
\hline Thrombozyten [Tausd/ $\mu$ l] & 97 & $150-450$ & C-reaktives Protein [mg/dl] & 0,28 & $<0,5$ \\
\hline International Normalized Ratio (INR) & 7,8 & $<1,2$ & Gesamteiweiß [g/dl] & 6,2 & $6,5-8,5$ \\
\hline Partielle Thrombin-Zeit (PTT) [sec.] & 140 & $<40$ & pH venös & 7,2 & $7,34-7,43$ \\
\hline Kreatinin [mg/dl] & 4,5 & $0,5-0,8$ & $\mathrm{pCO}_{2}$ & 37,2 & $35-45$ \\
\hline MDRD-GFR [ml/min/1,73 m²] & 9 & $>60$ & Standard-Bicarbonat [mmol/l] & 17,1 & $20-25$ \\
\hline Harnstoff [mg/dl] & 347 & $12-46$ & & & \\
\hline
\end{tabular}


DMW Falldatenbank

\section{Kasuistik | Diagnostik | Weitere Laboruntersuchungen}

\begin{tabular}{|l|c|l|}
\hline Parameter & Wert & \multicolumn{1}{c|}{ Referenzbereich } \\
\hline Digitoxin $[\mu \mathrm{g} / \mathrm{dl}]$ & 3,2 & $1,2-2,5$ (therapeutischer Bereich) \\
\hline Dabigatran $[\mathrm{ng} / \mathrm{ml}]^{1}$ & 2230 & Erwartete Werte: \\
\hline & & $117-275$ Spitzenspiegel $^{2}$ \\
\hline & & $61-143$ Talspiegel (TS) ${ }^{2}$ \\
\hline & & TS $>200$ erhöhtes Blutungsrisiko $^{2}$
\end{tabular}

${ }^{1}$ gemessen mit dem Hemoclot@-Assay

$>$ Abdomen-Sonographie:

$>$ Nieren altersentsprechend verkleinert, Harnblase leer, kein Harnstau

$>$ V. cava inferior komplett kollaptisch, kein Aszites, keine Pleuraergüsse

$>$ geringgradiger Perikardsaum 
DMW Falldatenbank

\section{Kasuistik | EKG}
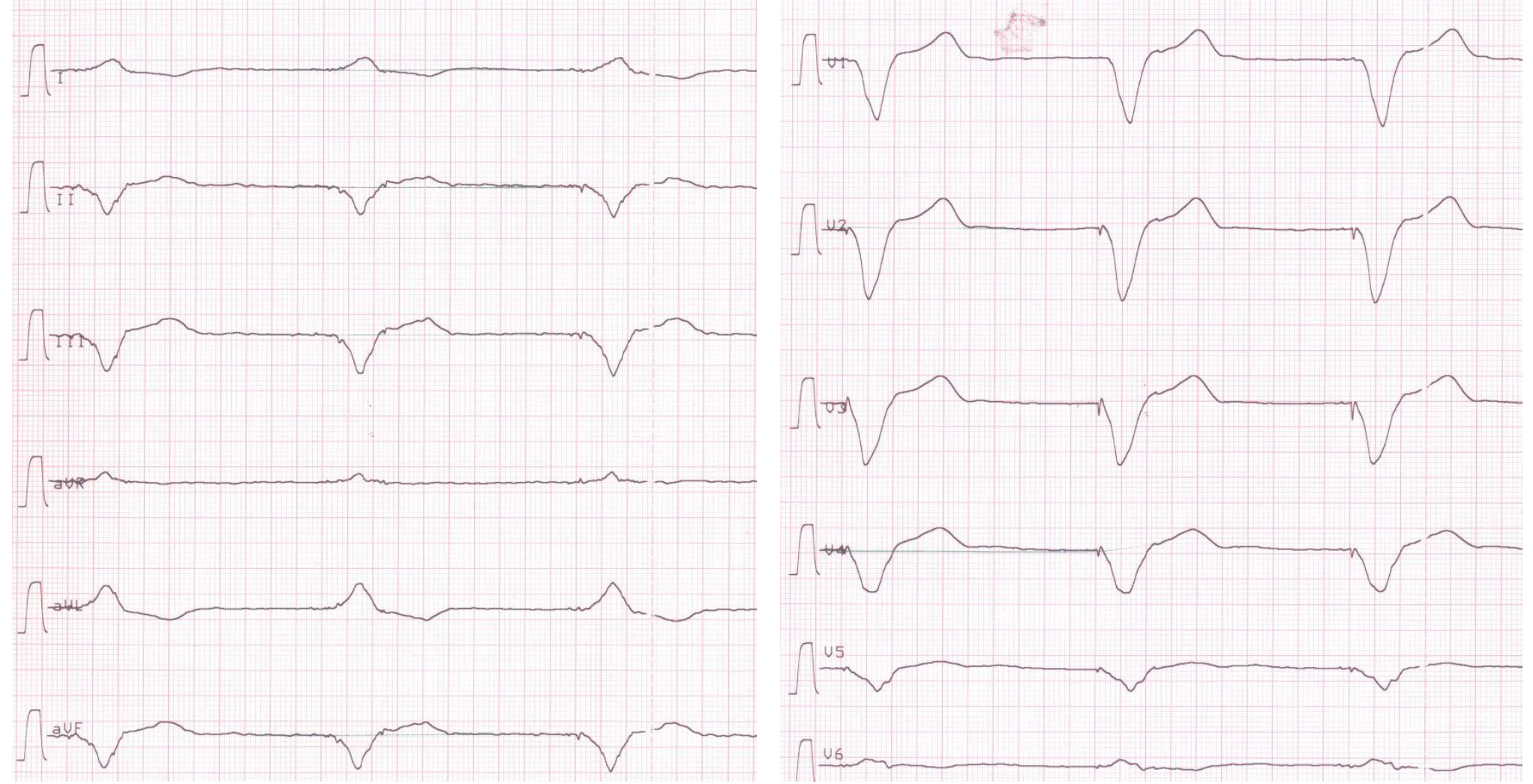

$10 \mathrm{~mm} / \mathrm{mV}$

$50 \mathrm{~mm} / \mathrm{s}$
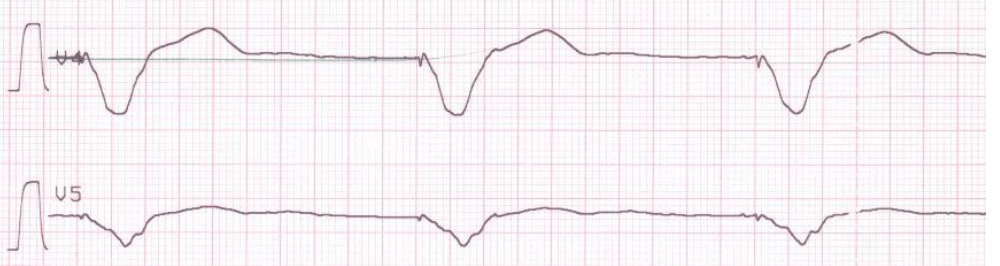

$>\quad$ Ihr Befund? 
DMW

\section{Falldatenbank}

\section{Kasuistik | EKG}
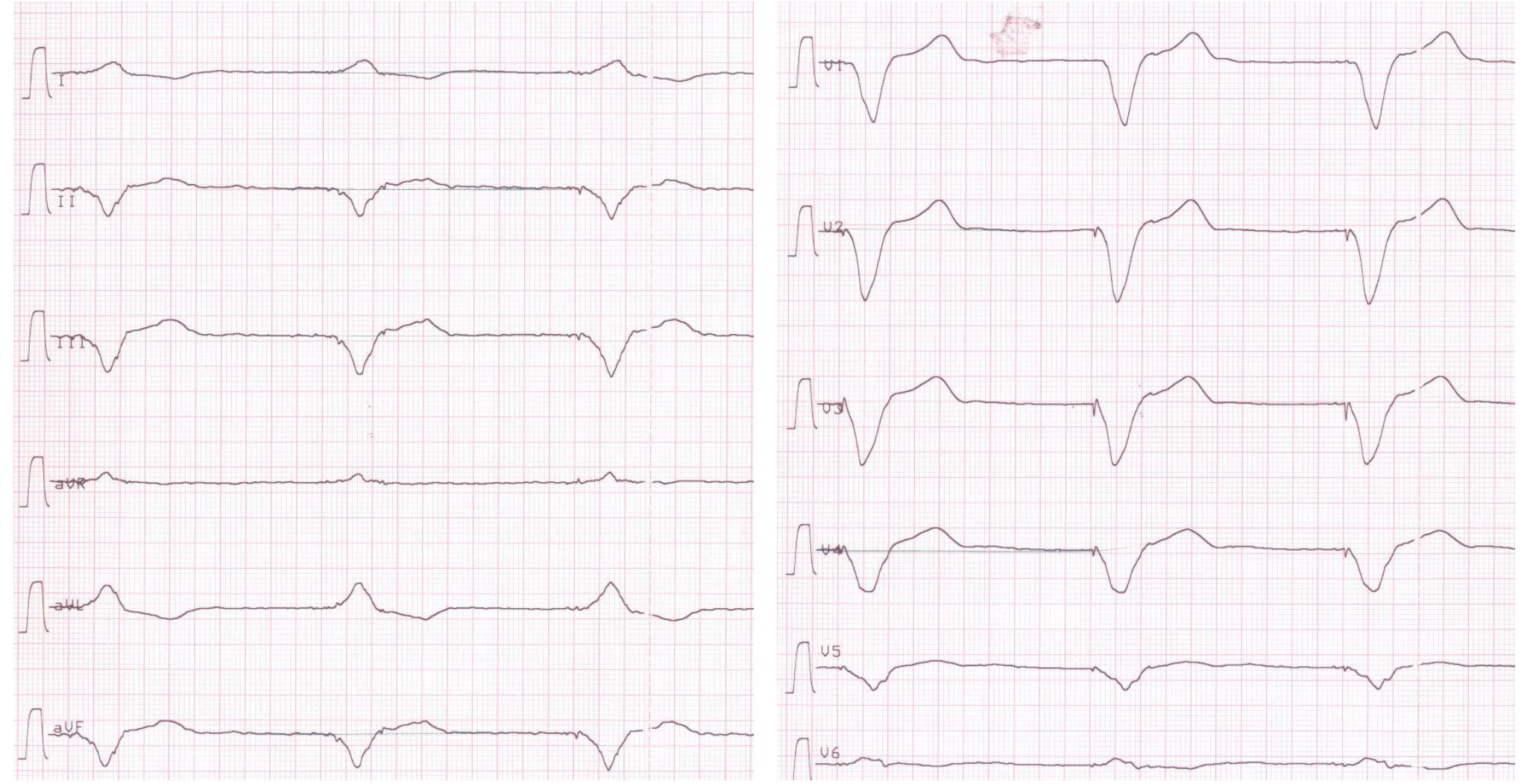

$10 \mathrm{~mm} / \mathrm{mV}$

$50 \mathrm{~mm} / \mathrm{s}$
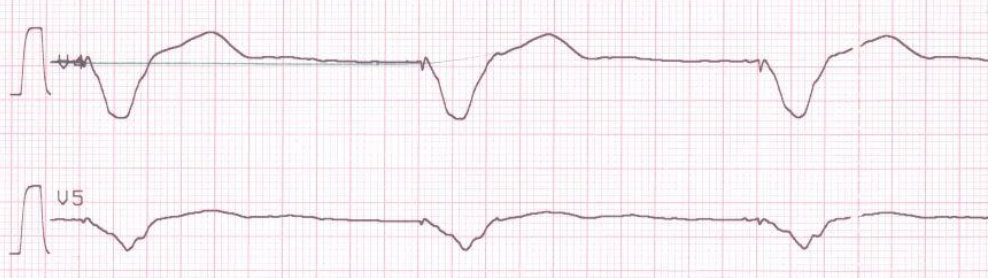

> Schrittmacher-EKG, ventrikuläre Stimulation, Herzfrequenz 60/min 


\section{Kasuistik | Diagnose}

> Vital gefährdende Dabigatran-Akkumulation bei akut-auf-chronischer Niereninsuffizienz mit Anurie infolge Diuretika-assoziierter Exsikkose 
DMW

\section{Falldatenbank}

\section{Kasuistik | Therapie}

$>$ Intravenöse Flüssigkeitsgabe (balancierte kristalloide Lösung [Jonosteril ${ }^{\circledR}$ ],

initial $3500 \mathrm{ml}$ in $6 \mathrm{~h}$ )

$>$ hierunter Einsetzen der Diurese

> Pausierung aller Medikamente (Diuretika, AT-Blocker, Digitoxin, Beta-Blocker, Dabigatran)

> Indikationsstellung zur Hämodialyse (HD) aufgrund der urämischen Stoffwechsellage (urämischer Fötor) und der Dabigatran-Kumulation mit veränderter Gerinnung

$>$ Substitution von Gerinnungsfaktoren vor Shaldon-Katheteranlage:

$>2 \mathrm{mg}$ rekombinater Faktor VIla

$>2000$ I.E. Prothrombinkomplex-Konzentrat (PPSB) bestehend aus Prothrombin (Faktor II), Prokonvertin (Faktor VII), Stuart-Prower-Faktor (Faktor X), antihämophiler Faktor B (Faktor IX)

> Komplikationslose Shaldon-Katheteranlage und Einleitung einer Hämodialyse (HD) 


\section{Kasuistik | Röntgen-Thorax}

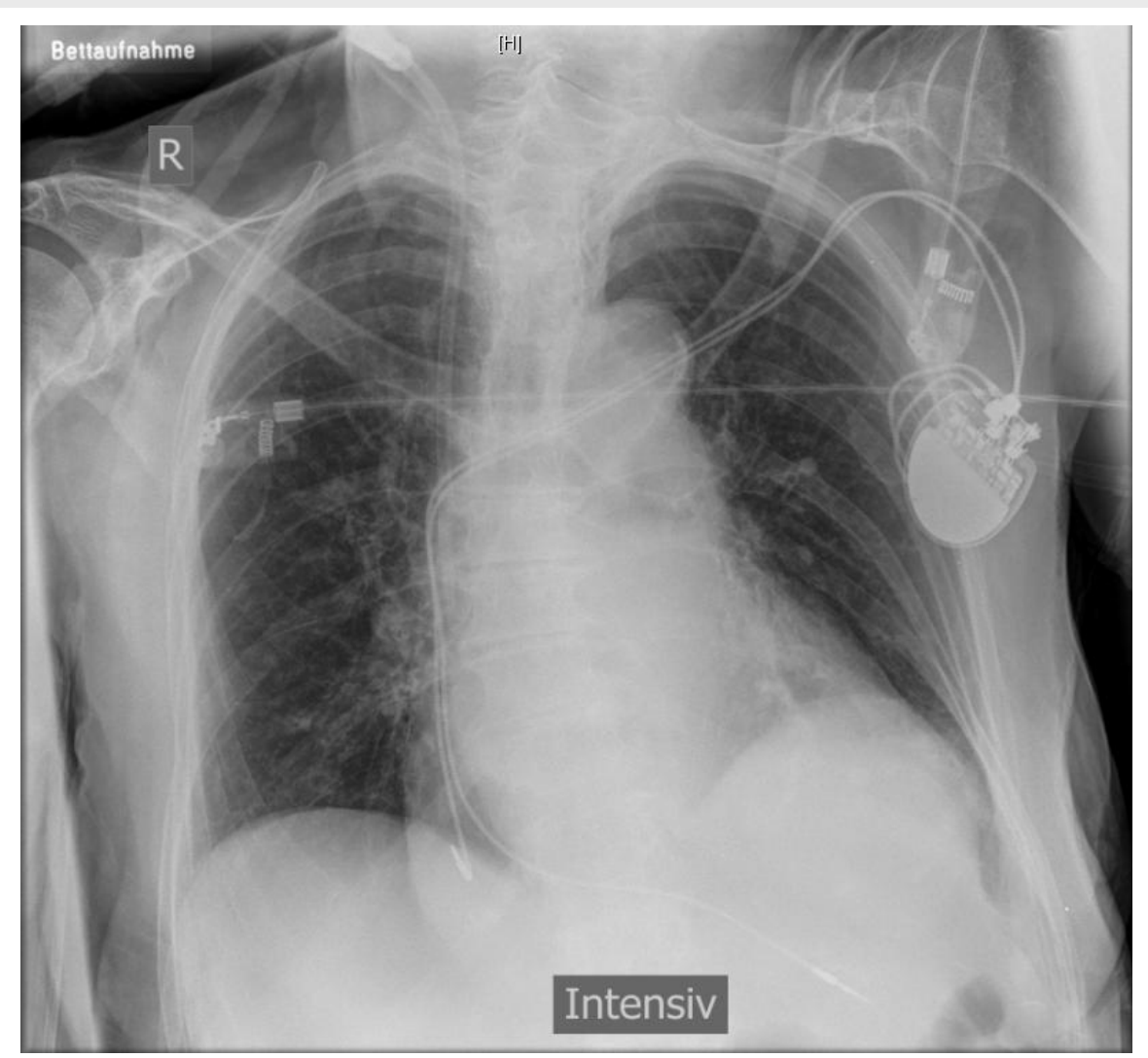

$>\quad$ Ihr Befund? 


\section{Kasuistik | Röntgen-Thorax}

$>$ Kardiomegalie ohne Stauungszeichen $\left({ }^{*}\right)$

$>$ Keine Belüftungsstörung oder Infiltrat

$>$ Shaldon-Katheter rechts jugulär in Höhe der Vena cava superior (weißer Pfeil)

$>$ Kein Pneumothorax

> Schrittmacheraggregat links pektoral mit konnektierenden Sonden in Projektion auf den rechten Ventrikel und den rechten Vorhof (blauer Pfeil)

> Rechtskonvexe Thorakalskoliose (roter Pfeil)

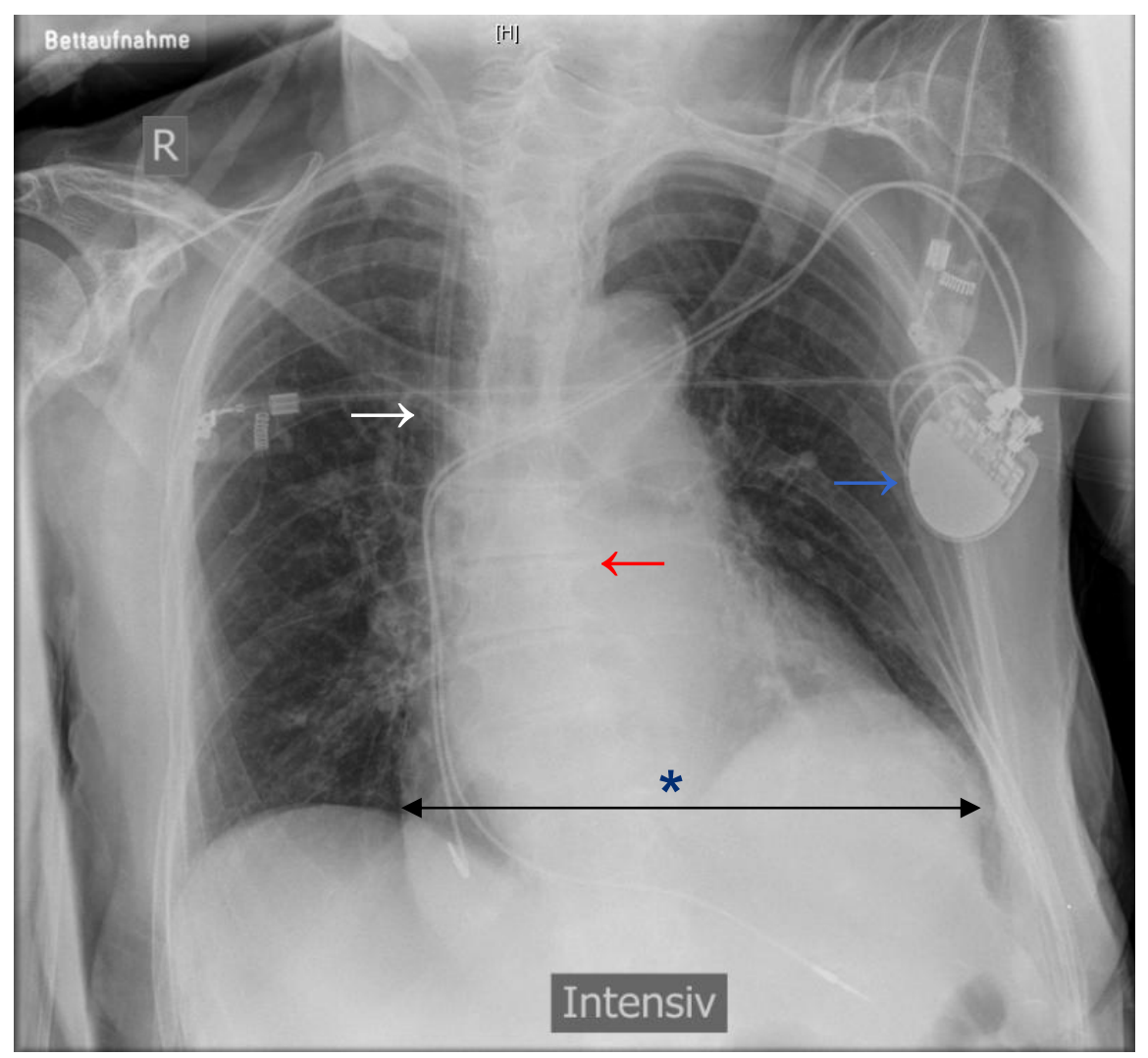


DMW

\section{Falldatenbank}

\section{Kasuistik | Therapie}

$>\quad$ Die erste HD erfolgte als SLED (sustained low efficiency dialysis) über Nacht mit einem Fresenius Genius 90-Gerät:

$>$ Dauer: 10 h, Blutfluss $150 \mathrm{ml} / \mathrm{min}$, Dialysatfluss $150 \mathrm{ml} / \mathrm{min}$, High-Flux-Dialysator

> Am Nachmittag des 1. Tages wurde eine Standard-HD mit einem Fresenius 4008S-Gerät durchgeführt:

$>$ Dauer: 3 h, Blutfluss 300 ml/min, Dialysatfluss 500 ml/min, High-Flux-Dialysator

> Nach 2 Tagen wurde eine Online-Hämodiafiltration (OL-HDF) mit einem Fresenius 5008-Gerät durchgeführt:

> Dauer: 4 h, Blutfluss 300 ml/min, Dialysatfluss 300 ml/min, Substituat 24 l, High-Flux-Dialysator

$>$ Die HD-Sitzungen erfolgten ohne zusätzliche Antikoagulation. 
DMW Falldatenbank

\section{Kasuistik | Kreatinin- und Dabigatran-Spiegelverlauf}

> Die Kreatinin- und Dabigatran-Plasma-Konzentrationen sanken unter Hämodialyse und einsetzender Nierenfunktion:

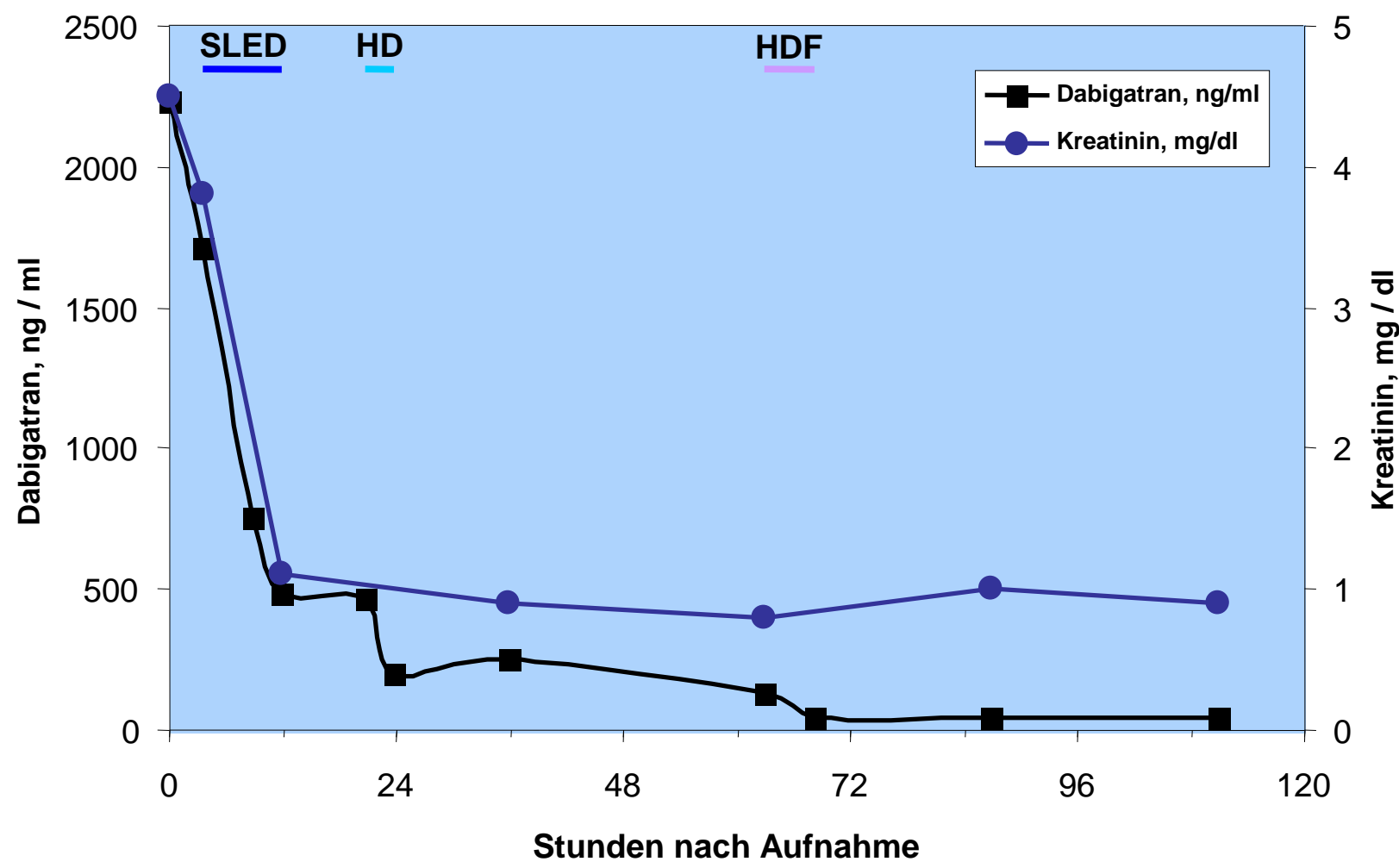

SLED: sustained low efficiency extended dialysis HD: Standard-Hämodialyse HDF: Hämodiafiltration

Stunden nach Aufnahme 
DMW Falldatenbank

\section{Kasuistik | Verlauf der Gerinnungstests}

> Mit Abfall der Dabigatran-Konzentration normalisierten sich die Gerinnungstests weitgehend:

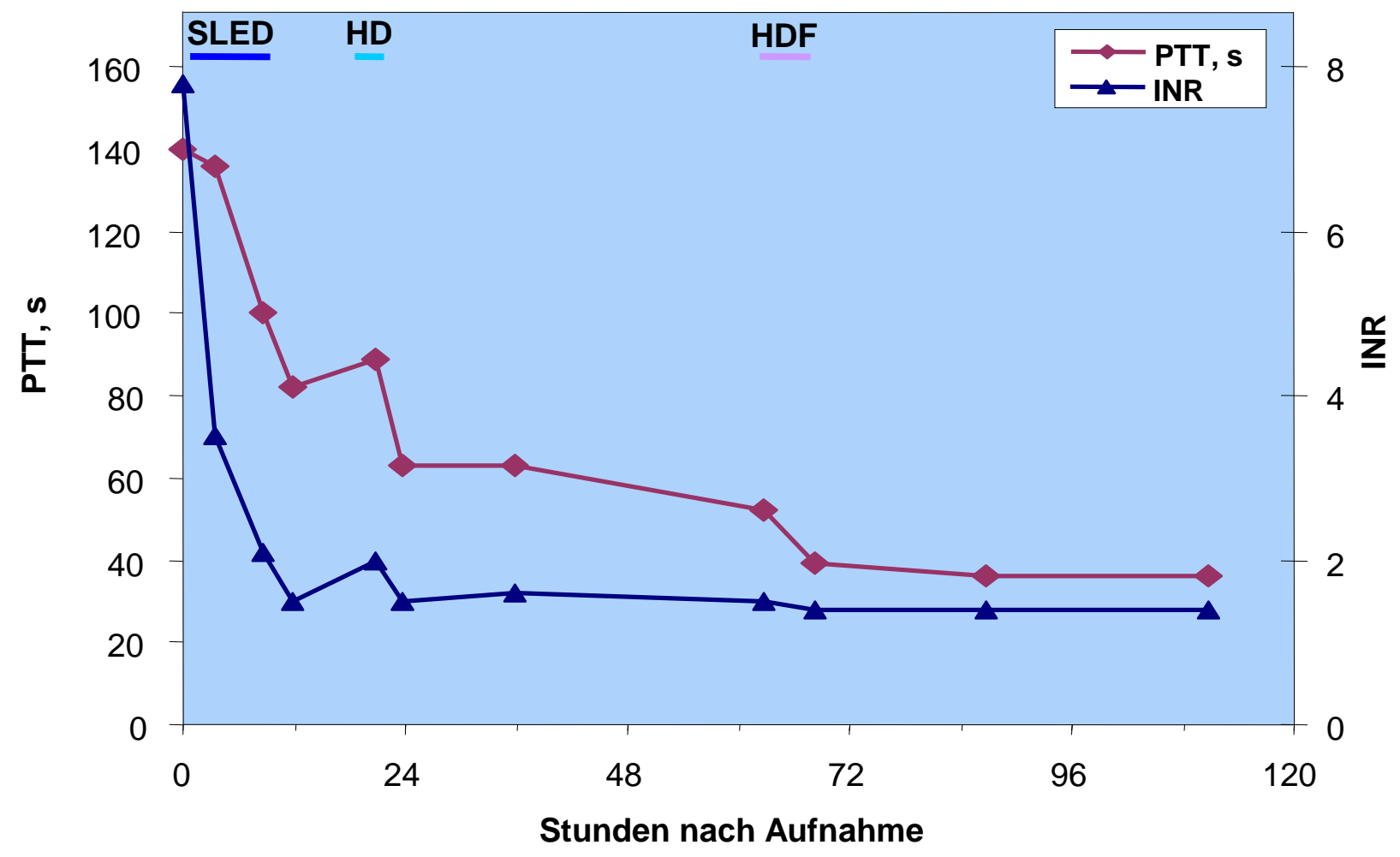

SLED: sustained low efficiency extended dialysis HD: Standard-Hämodialyse HDF: Hämodiafiltration 
Tübinger Fälle

\section{䀧Thieme}

\section{Kasuistik | Diagnostik | Labor an Tag 4 nach Aufnahme}

\begin{tabular}{|c|c|c|c|c|c|}
\hline Parameter & Wert & Referenzbereich & Parameter & Wert & Referenzbereich \\
\hline Leukozyten $[1 / \mu l]$ & 9440 & $4100-11800$ & Harnstoff [mg/dl] & 25 & $12-46$ \\
\hline Hämoglobin [g/dl] & 10,2 & $12,0-16,0$ & Natrium [mmol/l] & 143 & $136-148$ \\
\hline Thrombozyten [Tausd/ $\mu l]$ & 39 & $150-450$ & Kalium [mmol/l] & 3,7 & $3,5-4,8$ \\
\hline Kreatinin [mg/dl] & 1,1 & $0,6-0,8$ & Kalzium [mmol/l] & 2,2 & $2,1-2,3$ \\
\hline MDRD-GFR [ml/min/1,73 m²] & 47 & $>60$ & Phosphat [mmol/l] & 0,5 & $0,8-1,5$ \\
\hline Cystatin C [mg/l] & 2,3 & $0,5-1,0$ & $\begin{array}{l}\text { International Normalized } \\
\text { Ratio (INR) }\end{array}$ & 1,5 & $<1,2$ \\
\hline CKD-EPI-Cys-C-GFR [ml/min/1,73 m²] & 21 & $>60$ & $\begin{array}{l}\text { Partielle Thrombin-Zeit } \\
\text { (PTT) [sec.] }\end{array}$ & 39 & $<40$ \\
\hline \multicolumn{6}{|l|}{ 24-Stunden-Sammelurin } \\
\hline Volumen [ml] & 1550 & & Eiweiß [g pro 24 h] & 0,17 & $<0,15$ \\
\hline Kreatinin-Clearance [ml/min] & 30 & $>60$ & Albumin [mg pro $24 \mathrm{~h}$ ] & 29 & $<30$ \\
\hline $\mathrm{GFR}^{*}[\mathrm{ml} / \mathrm{min}]$ & 23 & $>60$ & & & \\
\hline
\end{tabular}

$>$ Ihr Befund? 


\section{Kasuistik | Diagnostik | Labor an Tag 4 nach Aufnahme}

\begin{tabular}{|c|c|c|c|c|c|}
\hline Parameter & Wert & Referenzbereich & Parameter & Wert & Referenzbereich \\
\hline Leukozyten $[1 / \mu l]$ & 9440 & $4100-11800$ & Harnstoff [mg/dl] & 25 & $12-46$ \\
\hline Hämoglobin [g/dl] & 10,2 & $12,0-16,0$ & Natrium [mmol/l] & 143 & $136-148$ \\
\hline Thrombozyten [Tausd/ $\mu l]$ & 39 & $150-450$ & Kalium [mmol/l] & 3,7 & $3,5-4,8$ \\
\hline Kreatinin [mg/dl] & 1,1 & $0,6-0,8$ & Kalzium [mmol/l] & 2,2 & $2,1-2,3$ \\
\hline MDRD-GFR [ml/min/1,73 m²] & 47 & $>60$ & Phosphat [mmol/l] & 0,5 & $0,8-1,5$ \\
\hline Cystatin C [mg/l] & 2,3 & $0,5-1,0$ & $\begin{array}{l}\text { International Normalized } \\
\text { Ratio (INR) }\end{array}$ & 1,5 & $<1,2$ \\
\hline CKD-EPI-Cys-C-GFR $\left[\mathrm{ml} / \mathrm{min} / 1,73 \mathrm{~m}^{2}\right]$ & 21 & $>60$ & $\begin{array}{l}\text { Partielle Thrombin-Zeit } \\
\text { (PTT) [sec.] }\end{array}$ & 39 & $<40$ \\
\hline \multicolumn{6}{|l|}{ 24-Stunden-Sammelurin } \\
\hline Volumen [ml] & 1550 & & Eiweiß [g pro 24 h] & 0,17 & $<0,15$ \\
\hline Kreatinin-Clearance [ml/min] & 30 & $>60$ & Albumin [mg pro $24 \mathrm{~h}$ ] & 29 & $<30$ \\
\hline $\mathrm{GFR}^{*}[\mathrm{ml} / \mathrm{min}]$ & 23 & $>60$ & & & \\
\hline
\end{tabular}

> Zunehmende Thrombopenie (am ehesten durch vermehrten Verbrauch), spontan eingeschränkte INR, deutliche Niereninsuffizienz, Überschätzung der GFR durch MDRD-Formel, Hypophosphatämie (Mangelernährung) 
DMW

\section{Falldatenbank}

\section{Kasuistik | Verlauf}

> Die vaginalen Blutungen gingen zurück, sistierten aber nicht vollständig.

Die Tamponaden mussten entsprechend mehrfach ausgetauscht werden.

$>$ Insgesamt wurden 5 Erythrozytenkonzentrate substituiert.

> Am 5.Tag wurde die Patientin zur vaginalen Hysterektomie in die Frauenklinik verlegt.

> Bei stabilem Zustand starb die Patientin 2 Tage vor dem geplanten Eingriff am plötzlichen Herztod durch Kammerflimmern. 


\section{Diskussion | Akut-auf-chronisches Nierenversagen}

> In vorliegenden Fall kam es zu einem akut-auf-chronischem Nierenversagen und in der Folge zu einer massiven Kumulation von Dabigatran auf mehr als das 20-Fache der Talspiegel.

$>$ Ursächlich dafür war ein Volumenmangel durch eine Diuretika-Therapie bei Herzinsuffizienz (prärenale Genese).

$>\quad$ Derartige Episoden von prärenalem Nierenversagen sind bei herzinsuffizienten Patienten sehr häufig [1].

$>$ In einer Studie aus der Schweiz zeigte sich eine Verschlechterung der Nierenfunktion bei bis zu 22\% aller herzinsuffizienten Patienten innerhalb von 6 Monaten.

$>$ Die Behandlung mit Schleifendiuretika oder Spironolacton war unabhängiger Risikofaktor.

$>\quad$ Bei höhergradiger Herzinsuffizienz besteht eine renale Minderperfusion, die zu einer GFR-Reduktion und chronischen Niereninsuffizienz führt und einem kardiorenalen Syndrom (Typ 2) entspricht [2,3].

$>$ Unter Volumenmangel kann schließlich die renale Minderperfusion so verschlechtert werden, dass der glomeruläre Filtrationsdruck und die Filtrationsrate kritisch abfallen und ein akut-aufchronisches Nierenversagen induziert wird. 


\section{Pharmakologie | Die neuen oralen Antikoagulanzien}

$>$ Zu den neuen oralen Antikoagulanzien (NOAK) gehören Dabigatran, Rivaroxaban und Apixaban [1].

> Sie zeichnen sich durch eine führende renale Elimination aus. Die Präparate sind daher bei Patienten mit höhergradiger Niereninsuffizienz kontraindiziert.

$>$ Patienten unter laufender Therapie benötigen kein Monitoring der Gerinnung.

$>$ Dabigatran wird als Prodrug aufgenommen und mittels Hydrolyse aktiviert, das Plasmaverteilungsvolumen beträgt ca. 60-70 I [2].

\begin{tabular}{|l|c|c|c|}
\hline & Dabigatran & Rivaroxaban & Apixaban \\
\hline Wirkmechanismus & Direkter Thrombin-Inhibitor & Faktor-Xa-Inhibitor & Faktor-Xa-Inhibitor \\
\hline Spitzenspiegel nach $(\mathrm{h})$ & $0,5-3$ & $2-4$ & $1-3$ \\
\hline Plasmaeiweißbindung (\%) & 35 & $92-95$ & 27 \\
\hline Renale Elimination (\%) & 85 & 66 & 25 \\
\hline Halbwertszeit $(\mathrm{h})$ & $12-17$ & $9-13$ & $10-14$ \\
\hline Kontraindikation & $\mathrm{CrCl}<30 \mathrm{ml} / \mathrm{min}$ & $\mathrm{CrCl}<30 \mathrm{ml} / \mathrm{min}$ & $\mathrm{CrCl}<15 \mathrm{ml} / \mathrm{min}$ \\
\hline
\end{tabular}


DMW Falldatenbank

\section{Pharmakologie | Dosierung von Dabigatran}

> Primärprävention einer tiefen Beinvenethrombose nach Gelenkersatz (Hüfte/Knie) [1]:

$>1 \times 220 \mathrm{mg} /$ Tag für 10 Tage bei Knieersatz bzw. 28-35 Tage nach Hüftersatz

$>$ bei eingeschränkter Nierenfunktion ( $\mathrm{CrCl} 30-50 \mathrm{ml} / \mathrm{min}) 1$ x $150 \mathrm{mg} / \mathrm{Tag}$

$>$ Prävention eines Schlaganfalls bei nicht-valvulärem Vorhofflimmern und einem

CHA2DS2-Vasc-Score $>1$ [1]

$>2 \times 150 \mathrm{mg} / \mathrm{Tag}$

$>$ bei eingeschränkter Nierenfunktion $(\mathrm{CrCl} 30-50 \mathrm{ml} / \mathrm{min})$ und einem erhöhten Blutungsrisiko $2 \times 110 \mathrm{mg} / \mathrm{Tag}$

$>$ Ein erhöhtes Blutungsrisiko besteht bei [2]

$>$ Alter $\geq 75$ Jahre

$>\mathrm{CrCl} 30-50 \mathrm{ml} / \mathrm{min}$

> gleichzeitiger Therapie mittels ASS, Clopidogrel, nicht-steroidaler Antiphlogistika

$>$ Kontraindikationen

$>$ akute Blutungen

$>$ Niereninsuffizienz mit $\mathrm{CrCl}<30 \mathrm{ml} / \mathrm{min}$

$>$ eingeschränkte Leberfunktion

> Therapie mittels Proteasehemmer, Ketokonazol, Ciclosporin, Itraconazol oder Tacrolimus (verminderter Abbau von Dabigatran durch P-Glykoprotein)

1 Rote Liste ${ }^{\circledR}$ 


\section{Pharmakologie | Dabigatran-Monitoring}

$>$ Grundsätzlich ist ein Gerinnungs-Monitoring nicht erforderlich [1].

> Die gebräuchlichen Gerinnungstests (wie PTT oder Quick) werden durch Dabigatran nur gering und nur während der Spitzenspiegel (1-3 Stunden nach Einnahme) verlängert, sie werden in der Regel am Ende des Dosisintervalls (Talspiegel) nicht verändert [2,3].

$>$ Hingegen ist die Verlängerung der PTT bei erhöhten Dabigatran-Konzentrationen nicht linear und erreicht ein Plateau [2].

$>$ Am sensitivsten wird die Dabigatran-Wirkung durch eine Verlängerung der Thrombinzeit (TZ) und die Ecarin-Clotting-Zeit erfasst [3,4].

$>$ Diese Tests sind jedoch im klinischen Alltag nicht allseits verfügbar.

> Mit der verdünnten TZ (dTZ; Hemoclot-Test ${ }^{\circledR}$, HYPHEN BioMed, Frankreich, Vertrieb in Deutschland durch CoaChrom Diagnostica GmbH) steht ein Test zur Verfügung, der die Wirkung direkter ThrombinInhibitoren messen kann (neben Dabigatran auch Argatroban und Hirudin) und das Ergebnis auf eine Dabigatran-Plasma-Konzentration zurückrechnet.

$>$ Die Verlängerung der TZ durch Dabigatran war über einen weiten Konzentrationsbereich von $0-1886 \mathrm{ng} / \mathrm{ml}$ linear [5].

$>$ In unserem Fall wurde die Dabigatran-Plasma-Konzentration ebenfalls mit dem Hemoclot ${ }^{\circledR}$-Test erfasst. 


\section{Pharmakologie | Dabigatran-Monitoring}

$>$ Für die Plasma-Dabigatran-Konzentration gibt es keine Ziel- oder Normwerte. Anhand der Dosierung sind folgende Plasma-Konzentration in Abhängigkeit von der Einnahme zu erwarten:

\begin{tabular}{|c|c|c|c|c|}
\hline & \multicolumn{2}{|c|}{$\begin{array}{l}\text { Dabigatran-Konzentration } \\
\text { im steady state }\end{array}$} & \multicolumn{2}{|c|}{ Blutungsrisiko } \\
\hline Dosis & $\begin{array}{l}\text { Spitzenspiegel } \\
\text { ( } 2 \text { h nach Einnahme) }\end{array}$ & Talspiegel & $\begin{array}{l}\text { wenn Talspiege } \\
\text { dTZ }\end{array}$ & $\begin{array}{l}>90 \text {. Perzentile } \\
\text { PTT }\end{array}$ \\
\hline $\begin{array}{l}150 \mathrm{mg} \text { alle } 12 \mathrm{~h} \\
\text { (Standarddosis) }\end{array}$ & $\begin{array}{l}175 \mathrm{ng} / \mathrm{ml} \\
117-275 \mathrm{ng} / \mathrm{ml} \\
(=25 .-75 . \text { Perzentile })\end{array}$ & $\begin{array}{l}91 \mathrm{ng} / \mathrm{ml}(\text { nach } 12 \mathrm{~h}) \\
61-143 \mathrm{ng} / \mathrm{ml} \\
(=25 .-75 . \text { Perzentile })\end{array}$ & $\begin{array}{l}>200 \mathrm{ng} / \mathrm{ml} \\
\text { (nach } 10-16 \mathrm{~h})\end{array}$ & $\begin{array}{l}>80 \mathrm{~s} \\
\text { d. h. }>2 \text {-fach } \\
\text { des oberen } \\
\text { Referenzwerts }\end{array}$ \\
\hline $110 \mathrm{mg}$ alle $12 \mathrm{~h}$ & \multicolumn{4}{|c|}{ keine Angabe: Verweis auf $150 \mathrm{mg}$ alle $12 \mathrm{~h}$} \\
\hline $2 \times 110 \mathrm{mg}$ alle $24 \mathrm{~h}$ & $\begin{array}{l}71 \mathrm{ng} / \mathrm{ml} \\
35-162 \mathrm{ng} / \mathrm{ml} \\
(=25 .-75 . \text { Perzentile })\end{array}$ & $\begin{array}{l}22 \mathrm{ng} / \mathrm{ml} \text { (nach } 24 \mathrm{~h}) \\
13-36 \mathrm{ng} / \mathrm{ml} \\
(=25 .-75 . \text { Perzentile) }\end{array}$ & $\begin{array}{l}>67 \mathrm{ng} / \mathrm{ml} \\
\text { (nach } 20-28 \mathrm{~h})\end{array}$ & $\begin{array}{l}>51 \mathrm{~s} \\
\text { d. } \mathrm{h} .>1,3 \text {-fach } \\
\text { des oberen } \\
\text { Referenzwerts }\end{array}$ \\
\hline $2 \times 75 \mathrm{mg}$ alle $24 \mathrm{~h}$ & \multicolumn{4}{|c|}{ keine Angabe: Verweis auf $2 \times 110 \mathrm{mg}$ alle $24 \mathrm{~h}$} \\
\hline
\end{tabular}




\section{Pharmakologie | Abschätzung der Nierenfunktion}

$>$ Die Abschätzung der Nierenfunktion ist vor Beginn einer Therapie mit den neuen oralen Antikoagulanzien von größter Bedeutung.

> Dies kann einerseits durch die errechnete GFR geschehen, die aus der Plasma-KreatininKonzentrationen und auf weitere Variablen wie Alter oder Geschlecht zurückgreifen.

$>$ Am bekanntesten ist MDRD-Formel [1], deren vereinfachte Form am weitesten verbreitet ist.

$>$ Die CKD-EPI-Formel [2] hat Vorteile im Grenzbereich zur eingeschränkten Nierenfunktion (GFR 60 - 90 ml/min). Ab dem Stadium 3 besteht kein Unterschied zu MDRD-Formel.

$>$ Alternativ kann die Cystatin-C-Plasma-Konzentration bestimmt und daraus eine GFR errechnet werden.

$>$ Sie ist vorteilhaft bei Älteren und insbesondere kachektischen Patienten (geringere Muskelmasse und falsch hohe Einschätzung der GFR anhand Kreatinin-basierter Methoden).

$>$ Die Bestimmung der Kreatinin-Clearance aus einem Sammelurin hat folgende Einschränkungen:

$>$ Sie überschätzt die GFR durch tubuläre Sekretion von Kreatinin, was mit zunehmender Niereninsuffizienz weiter zunimmt.

$>$ Durch Sammelfehler wird die GFR hingegen unterschätzt.

$>$ Bei höhergradiger Niereninsuffizienz kommt der Mittelwert aus Kreatinin- und Harnstoff-Clearance der GFR am nächsten. 
DMW

\section{Falldatenbank}

KLINIKUM

TÜBINGEN

Tübinger Fälle

\section{Thieme}

\section{Diskussion | Tücken der Dabigatrantherapie -1-}

> Die Gerinnung der Patienten unter laufender Therapie mit NOAK wird nicht überwacht, da die Talspiegel nicht mit den üblichen Globaltest (PTT, INR) erfasst werden und dies nur von bestimmten Tests geleistet wird.

> Liegt jedoch eine signifikante Verlängerung der PTT (auf mehr als das 1,5-Fache) oder eine INR-Erhöhung vor, ist von einer Überdosierung bzw. Kumulation mit erhöhtem Blutungsrisiko auszugehen.

$>$ Im vorliegenden Fall betrug die PTT 140 s (entsprechend einer 3,5-fachen Verlängerung), die INR 7,8! 
DMW

\section{Falldatenbank}

UNIVERSITÄTS

KLINIKUM

TÜBINGEN

Tübinger Fälle

\section{格 Thieme}

\section{Diskussion | Tücken der Dabigatrantherapie -2-}

> Eine Kumulation von Dabigatran infolge Niereninsuffizienz ist ein lebensgefährlicher Zustand und war nach Markteinführung mit einer Reihe von Todesfällen assoziiert [1].

> Dieser Fall verdeutlicht, dass das Monitoring der Nierenfunktion von ebenso großer Bedeutung ist.

> Die europäische kardiologische Leitlinie von 2013 empfiehlt eine Kontrolle der Nierenfunktion alle 6 Monate bei Niereninsuffizienz Stadium $3(\mathrm{CrCl} 30-60 \mathrm{ml} / \mathrm{min})$, Alter > 75 Jahre oder reduziertem Allgemeinzustand/Gebrechlichkeit (,frailty“) [2].

$>$ Bei Kumulation von Dabigatran kann die Wirkung nicht antagonisiert werden und es existiert kein Antidot:

$>$ Die einzige Option ist die rasche Entfernung der Substanz durch die Wiederherstellung der Nierenfunktion und/oder Hämodialyse [3].

$>$ In Entwicklung befindet sich ein Dabigatran-bindender Antikörper (Fab-Fragment) [4]. 
DMW

\section{Falldatenbank}

KLINIKUM

TÜBINGEN
Tübinger Fälle

\section{Diskussion | Tücken der Dabigatrantherapie -3-}

> Im Notfall wird vom Hersteller bei aktiven Blutungen die Gabe eines Prothrombinkomplex-Konzentraten (PPSB) und/oder rekombinanter aktivierter Faktor VIla angeführt [1,2], allerdings ist ein therapeutischer Effekt in Anwesenheit eines Inhibitors fraglich.

> Wenn man sich zur Hämodialyse entscheidet, sollte man sich des Risiko der Dialysekatheteranlage bewusst sein, denn die Punktion ist bei ungerinnbarer Situation lebensgefährlich.

$>$ In der EHEC-HUS-Epidemie in Deutschland 2011 gingen 25\% der Todesfälle auf Blutungskomplikationen nach Dialysekatheteranlage zurück, dabei wiesen die Patienten oft eine schwere Thrombopenie auf [3]. 


\section{Diskussion | Dabigatran-Elimination mittels Hämodialyse}

> In unserem Fall wurden drei verschiedene Hämodialyse (HD)-Verfahren eingesetzt:

$>$ eine Standard-HD (Dauer: 3 h, Blutfluss 300 ml/min, Dialysatfluss 500 ml/min, High-Flux-Dialysator)

$>$ eine SLED (sustained low efficiency dialysis; Dauer: 10 h, Blutfluss 150 ml/min, Dialysatfluss $150 \mathrm{ml} / \mathrm{min}$, High-Flux-Dialysator)

> sowie eine Online-Hämodiafiltration (OL-HDF; Dauer: 4 h, Blutfluss 300 ml/min, Dialysatfluss 300 ml/min, Substituat 24 I, High-Flux-Dialysator)

$>$ Am effektivsten schnitt die SLED ab, bei der Standard-HD kam es zu einem Rebound.

$>$ Dies kann durch das große Verteilungsvolumen von Dabigatran erklärt werden.

$>\quad$ In einer systematischen Studie an HD-Patienten war die Dialysedauer der effektivste Eliminationsparameter [1].

> Untergeordnet waren Eigenschaften des Dialysators oder eingestellte Flussraten. 


\section{Diskussion | Fazit}

> Bei korrekter Indikationsstellung und unter Berücksichtigung der Anwendungseinschränkungen ist Dabigatran als sicheres Medikament zu werten.

$>$ Die Indikationsstellung bei speziellen Kollektiven ( $>75$ Jahre, Kachexie) sollte Beachtung finden.

$>$ Eine Cystatin-C-Bestimmung kann bei grenzwertiger Nierenfunktion Klarheit bringen.

$>$ Bei Niereninsuffizienz ist eine häufigere Überprüfung der Nierenfunktion als die einmal jährlich empfohlene sinnvoll.

$>$ Insbesondere sollte die Nierenfunktion in Situationen überprüft werden, in der eine Abnahme vermutet wird (z.B. Volumenmangel, reduzierte renale Perfusion).

> Eine Hämodialyse, die wie in diesem Fall über einen lange Zeitraum erfolgt, kann das Dabigatran eliminieren, was bei den anderen NOAK (Apixaban / Rivaroxaban) nicht möglich ist.

$>$ Dabei muss das Blutungsrisiko bei Anlage des Dialysekatheters abgewogen werden. 


\section{Zusammenfassung}

Anamnese und klinischer Befund: Eine 90-jährige Patientin, die wegen Vorhofflimmern Dabigatran erhielt, wurde mit spontaner vaginaler Blutung bei Endometrium-Karzinom notfallmäßig aufgenommen.

Untersuchungen: Im Labor fand sich ein akut-auf-chronisches Nierenversagen bei deutlichem Anstieg der Retentionsparameter. Die partielle Thromboplastinzeit (PTT) war auf das 3,5-fache verlängert, die International Normalized Ratio (INR) betrug 7,8. Dabigatran war massiv kumuliert (gemessene PlasmaKonzentration von $2230 \mathrm{ng} / \mathrm{ml})$.

Diagnose, Therapie und Verlauf: Die vaginalen Blutungen wurden mittels Tamponaden versorgt. Aufgrund der Urämie und der Dabigatran-Kumulation wurde eine Hämodialysebehandlung als SLED (sustained low efficiency dialysis) über Nacht durchgeführt. Unter Volumensubstitution konnte die Nierenfunktion rasch wiederhergestellt werden, so dass von einer prärenalen Genese ausgegangen wurde. Nach weiteren zwei Hämodialyse-Sitzungen war die Dabigatran-Konzentration nicht mehr messbar. Dabei normalisierten sich die Gerinnungstests parallel zum Rückgang der Dabigatran-Konzentration.

Folgerung: Dabigatran kann bei Entwicklung eines akuten Nierenversagens massiv kumulieren und eine lebensgefährliche Blutungsneigung induzieren. Mittels langsamer Hämodialyse (z.B. als SLED) kann Dabigatran effektiv entfernt werden.

Schlüsselwörter: Dabigatran - Nierenversagen - Hämodialyse - SLED 
DMW

\section{Falldatenbank}

UNIVERSITÄTS

KLINIKUM

TÜBINGEN

Tübinger Fälle

\section{Zusammenfassung | Abstract}

History and admission findings: A 90-year-old female patient treated with dabigatran for atrial fibrillation presented emergently with a spontaneous vaginal bleeding due to endometrium carcinoma.

Investigations: Laboratory analysis revealed azotemia consistent with acute-on-chronic renal failure. Coagulation was deranged (prolongation of activated partial thromboplastin time (aPTT) by 3.5-fold, international normalized ratio (INR) 7.8) due to a massive accumulation of dabigatran (measured plasma concentration $2230 \mathrm{ng} / \mathrm{ml}$ ).

Diagnosis, treatment and course: Vaginal bleedings were treated with external tamponade. Hemodialysis treatment was commenced due to uremia and dabigatran accumulation. Over night, the patient was dialysed with a SLED (sustained low efficiency dialysis) regimen. After volume resuscitation renal function promptly ensued indicating prerenal azotemia as a cause of renal failure. After two more hemodialysis sessions dabigatran concentrations were no longer detecable and this was paralleled by normalization of coagulation tests.

Conclusions: Dabigatran can accumulate massively during acute renal failure and evoke a life-threatening bleeding diathesis. Dabigatran can be removed with low efficient dialysis (such as SLED).

Keywords: dabigatran - renal failure - hemodialysis - SLED 
DMW Falldatenbank

\section{Diskussion zum Fall}

$>$ Diskussionsbeitrag erstellen

$>$ Diskussion ansehen

$>$ Weitere E-Fälle finden Sie unter http://www.thieme.de/dmw/

\section{Autorenerklärung}

Die Autoren erklären, dass sie keine finanzielle Verbindung mit einer Firma haben, deren Produkt in diesem Beitrag eine Rolle spielt (oder mit einer Firma, die ein Konkurrenzprodukt vertreibt).

\section{Korrespondenzadresse}

Prof. Dr. med. Ferruh Artunc

Innere Medizin IV, Sektion Nieren- und Hochdruckkrankheiten

Universitätsklinikum Tübingen

Otfried-Müller-Str. 10

72076 Tübingen

Tel.: 07071/29 82711

Fax: 07071/29 5705

E-Mail: ferruh.artunc@med.uni-tuebingen.de

\section{Bibliografie}

DOI 10.1055/s-0034-1370191

๑Georg Thieme Verlag KG • Stuttgart New York

Impressum 